\title{
Proliferation and motility of hepatocellular, pancreatic and gastric cancer cells grown in a medium without glucose and arginine, but with galactose and ornithine
}

\author{
MINORU TOMIZAWA ${ }^{1}$, FUMINOBU SHINOZAKI ${ }^{2}$, YASUFUMI MOTOYOSHI ${ }^{3}$, \\ TAKAO SUGIYAMA ${ }^{4}$, SHIGENORI YAMAMOTO ${ }^{5}$ and NAOKI ISHIGE ${ }^{6}$ \\ Departments of ${ }^{1}$ Gastroenterology, ${ }^{2}$ Radiology, ${ }^{3}$ Neurology, ${ }^{4}$ Rheumatology, ${ }^{5}$ Pediatrics and \\ ${ }^{6}$ Neurosurgery, National Hospital Organization, Shimoshizu Hospital, Yotsukaido, Chiba 284-0003, Japan
}

Received August 4, 2015; Accepted November 10, 2016

DOI: $10.3892 / \mathrm{ol} .2017 .5568$

\begin{abstract}
Human primary hepatocytes are able to survive in a medium without glucose and arginine, but supplemented with galactose and ornithine (hepatocyte selection medium; HSM). To address the possibility of the application of HSM in cancer therapy, hepatocellular carcinoma cells, pancreatic cancer cells and gastric cancer cells were cultured in HSM. Cell proliferation was analyzed using an MTS assay. Morphological changes were analyzed using hematoxylin and eosin staining. Apoptosis was analyzed using a TUNEL assay and cell motility was assessed with a scratch assay. Cell proliferation was significantly suppressed in cell lines grown in HSM $(\mathrm{P}<0.01$ in all the cell lines). Hematoxylin and eosin staining revealed pyknotic nuclei, suggesting that these cells had undergone apoptosis. The number of TUNEL-positive cells was significantly increased in HSM. In the scratch assay, the distance between the growing edge and the scratched edge was significantly lower $(\mathrm{P}<0.01$ in all the cell lines) in cells cultured in HSM, compared with those grown in Dulbecco's modified Eagle's medium or RPMI-1640. Therefore, the proliferation and motility of hepatocellular carcinoma cells, pancreatic cancer cells and gastric cancer cells was suppressed, and these cells subsequently underwent apoptosis in a medium without glucose and arginine, but containing galactose and ornithine.
\end{abstract}

\section{Introduction}

The liver is a target organ implicated in a number of primary and metastatic types of cancer. Primary liver cancer types

Correspondence to: Dr Minoru Tomizawa, Department of Gastroenterology, National Hospital Organization, Shimoshizu Hospital, 934-5 Shikawatashi, Yotsukaido, Chiba 284-0003, Japan E-mail: nihminor-cib@umin.ac.jp

Key words: glucose, arginine, galactose, ornithine, galactokinase, urea cycle include hepatocellular carcinoma and cholangiocarcinoma; the most frequent original sites of metastasis in liver cancer are the stomach, pancreas and colon (1). Surgery is the most effective curative treatment for primary and metastatic liver cancer $(2,3)$; however, if surgery is not a viable option, chemotherapy or molecular target therapy are considered for further treatment (4-6). The agents used in chemotherapy and molecular targeted therapy frequently cause hepatotoxicity, which limits the efficacy of this treatment $(7,8)$. Therefore, the development of therapeutic approaches with a lower risk of hepatotoxicity is required.

Metabolism in cancer cells is characterized by increased aerobic glycolysis and lactate production, even under a sufficient oxygen supply (the Warburg effect), and cancer cells require more glucose compared with the surrounding normal tissues (9). This phenomenon is applied to positron emission tomography, in which 18-fluorodeoxyglucose, an analogue of glucose, is taken up by cancer cells (10). Unlike glucose, 18-fluorodeoxyglucose is not metabolized and accumulates in cancer cells; therefore, a positive signal may be detected from cancerous tissues (11). Glucose is an important source of energy that is essential for cell survival $(12,13)$. Galactose enters glycolysis as a substrate for the enzyme galactokinase (GALK), which is expressed in the liver and kidney $(14,15)$. Arginine is an essential amino acid produced from ornithine by ornithine carbamoyltransferase (OTC) in the urea cycle, which is exclusive to hepatocytes (16). Normal cells produce arginine de novo, whereas cancer cells take up arginine from extracellular tissues (17).

Hepatocytes express GALK and OTC, and therefore, may be expected to survive in a medium lacking glucose and arginine, but supplemented with galactose and ornithine $(18,19)$. Our previous study developed a hepatocyte selection medium (HSM), which lacks glucose and arginine but contains galactose and ornithine (20). Primary human hepatocytes are able to survive in HSM, and this medium purifies primary human hepatocytes from co-culture with human-induced pluripotent stem cells (21).

Therefore, the present study analyzed the suppression of the proliferation and motility of hepatocellular carcinoma cells, pancreatic cancer cells and gastric cancer cells in HSM. 


\section{Materials and methods}

Cell culture. Human hepatocellular carcinoma HLF and $\mathrm{PLC} / \mathrm{PRF} / 5$ cells, human pancreatic cancer MIA-Paca2 and PANC-1 cells and human gastric cancer MKN45 and MKN74 cells were purchased from the Riken Cell Bank (Cell Engineering Division, Riken Biosource Center, Tsukuba, Japan). HLF cells, PLC/PRF/5 cells and MIA-Paca 2 cells were cultured in Dulbecco's modified Eagle's medium (DMEM; Sigma-Aldrich; Merck Millipore, Darmstadt, Germany) supplemented with $10 \%$ fetal bovine serum (FBS; Thermo Fisher Scientific, Inc., Waltham, MA, USA). PANC-1, MKN45 and MKN74 cells were cultured in RPMI-1640 (Sigma-Aldrich; Merck Millipore) supplemented with $10 \%$ FBS. Cell lines were cultured with $5 \%$ carbon dioxide at $37^{\circ} \mathrm{C}$ in a humidified chamber, and passaged twice a week.

HSM. HSM was prepared from amino acid powders, using the formulation method of Leibovitz-15 medium (Thermo Fisher Scientific, Inc.), but omitting arginine, tyrosine, glucose and sodium pyruvate, and adding galactose $(900 \mathrm{mg} / \mathrm{l}$; Wako Pure Chemical Industries, Ltd., Osaka, Japan), ornithine (1 mM; Wako Pure Chemical Industries, Ltd.), glycerol (5 mM; Wako Pure Chemical Industries, Ltd.) and proline (260 mM; Wako Pure Chemical Industries, Ltd.). Proline is necessary for DNA synthesis, and therefore, it was included in the medium (30 mg/l) (22). Knockout serum replacement (KSR; Thermo Fisher Scientific, Inc.) was used at a final concentration of $10 \%$ in place of FBS in order to establish defined xeno-free conditions in HSM.

Cell proliferation analysis. HLF, PLC/PRF/5, MIA-Paca2, PANC-1, MKN45 and MKN74 cells were trypsinized, harvested and seeded onto 96-well flat-bottomed plates at a density of 1,000 cells/well, then incubated at $37^{\circ} \mathrm{C}$ for $24 \mathrm{~h}$ in DMEM or RPMI-1640 supplemented with 10\% FBS. Subsequent to changing the medium to HSM, the cells were cultured at $37^{\circ} \mathrm{C}$ for a further $72 \mathrm{~h}$, and subjected to an MTS assay (Promega Corporation, Madison, WI, USA), according to the manufacturer's protocol. MTS is reduced by the cells into a colored formazan product that reduces the absorbance at $490 \mathrm{~nm}$; the absorbance at $490 \mathrm{~nm}$ was evaluated using an iMark Microplate Absorbance Reader (Bio-Rad Laboratories, Inc., Hercules, CA, USA).

Hematoxylin and eosin staining. HLF, PLC/PRF/5, MIA-Paca2, PANC-1, MKN45 and MKN74 cells were cultured in chamber slides (Matsunami, Kishiwada, Japan) in a $5 \% \mathrm{CO}_{2}$ atmosphere at $37^{\circ} \mathrm{C}$ in a humidified chamber, and subjected to hematoxylin and eosin staining (Muto Glass, Tokyo, Japan). The cells were fixed in $10 \%$ formalin at room temperature for $10 \mathrm{~min}$. The cells were stained with hematoxylin, and incubated at room temperature for $4 \mathrm{~min}$. The slides were incubated in water at $25^{\circ} \mathrm{C}$ for $30 \mathrm{~min}$. The cells were dehydrated in $100 \%$ ethanol for 10 min three times, and were stained with eosin for $4 \mathrm{~min}$. The cells were dehydrated in $100 \%$ ethanol for 10 min three times, and mounted under cover slips. The slides were observed under x200 magnification using an AX80 microscope (Olympus Corporation, Tokyo, Japan). Five different fields of view were observed.
TUNEL staining. Cells were cultured in chamber slides with $5 \%$ carbon dioxide at $37^{\circ} \mathrm{C}$ in a humidified chamber and apoptotic cells were detected using the Wako Apoptosis In Situ Detection kit (Wako Pure Chemical Industries, Ltd.), following the manufacturer's protocol. Apoptotic cells were analyzed using a TUNEL assay, which consists of the addition of TdT to the 3'-terminus of apoptotically fragmented DNA, followed by immunochemical detection using an anti-fluorescein antibody conjugated with horseradish peroxidase and diaminobenzidine (DAB) as the substrate, following the manufacturer's protocol. The stained slides were observed under x100 magnification using an AX80 microscope.

Scratch assay. HLF, PLC/PRF/5, MIA-Paca2, PANC-1, MKN45 and MKN74 cells were plated on 4-well chamber slides, and upon reaching $100 \%$ confluency, the cell monolayer was scratched with a sterile razor, incubated at $37^{\circ} \mathrm{C}$ for $48 \mathrm{~h}$ and stained with hematoxylin and eosin. The stained slides were observed under x200 magnification using an AX80 microscope. The distance between the scratched and growing edges of the cells was evaluated at five different fields.

Statistical analysis. Data were presented as the mean \pm standard deviation. Cell proliferation, TUNEL assay and scratch assay data were analyzed by one-way analysis of variance. Statistical analysis was performed using JMP 5.0J software (SAS Institute, Inc., Cary, NC, USA). P $<0.05$ was considered to indicate a statistically significant difference.

\section{Results}

To examine the effect of culture in HSM on cell proliferation, an MTS assay was performed following three days of culture in DMEM, RPMI-1640 or HSM (Fig. 1). Proliferation of HLF, PLC/PRF/5, MIA-Paca2, PANC-1, MKN45 and MKN74 cells cultured in HSM decreased to $23.4 \pm 5.9,15.7 \pm 7.0,24.0 \pm 10.4$, $19.0 \pm 10.3,25.3 \pm 9.3$ and $34.7 \pm 13.2 \%$, respectively. The results revealed that cell proliferation was significantly suppressed in HSM for all cell lines $(\mathrm{P}<0.01)$.

To observe morphological changes, hematoxylin and eosin staining was performed following two days of culture in HSM (Fig. 2). HLF cells (Fig. 2A), PLC/PRF/5 cells (Fig. 2B), MIA-Paca2 cells (Fig. 2C), PANC-1 cells (Fig. 2D), MKN45 cells (Fig. 2E) and MKN74 cells (Fig. 2F) all exhibited pyknotic nuclei, suggesting that the cells had undergone apoptosis.

To further examine whether HSM-induced apoptosis had occurred, a TUNEL assay was performed (Fig. 3). TUNEL-positive cells were observed in all cell lines (Fig. 3A). The percentage of apoptotic cells in HLF, PLC/PRF/5, MIA-Paca2, MKN45 and MKN74 cells cultured in DMEM or RPMI-1640 was $0.1 \pm 0.0,0.2 \pm 0.0,1.1 \pm 0.0,1.3 \pm 0.0,10.9 \pm 2.1$ and $0.1 \pm 0.0 \%$, respectively. The percentage of cells in HLF, PLC/PRF/5, MIA-Paca2, PANC-1, MKN45 and MKN74 cells cultured in HSM increased to $40.0 \pm 12.3,11.5 \pm 3.2,25.0 \pm 4.3$, $13.7 \pm 2.3,15.5 \pm 3.4$ and $6.3 \pm 1.4 \%$, respectively. The number of TUNEL-positive cells was significantly higher in cells cultured in HSM ( $\mathrm{P}<0.01$ in HLF, PLC/PRF/5, MIA-Paca2, PANC-1 and MKN74 cells; $\mathrm{P}=0.02$ in MKN45 cells) (Fig. 3B).

To investigate whether HSM suppresses cell motility, a scratch assay was performed (Fig. 4). Each cell monolayer 


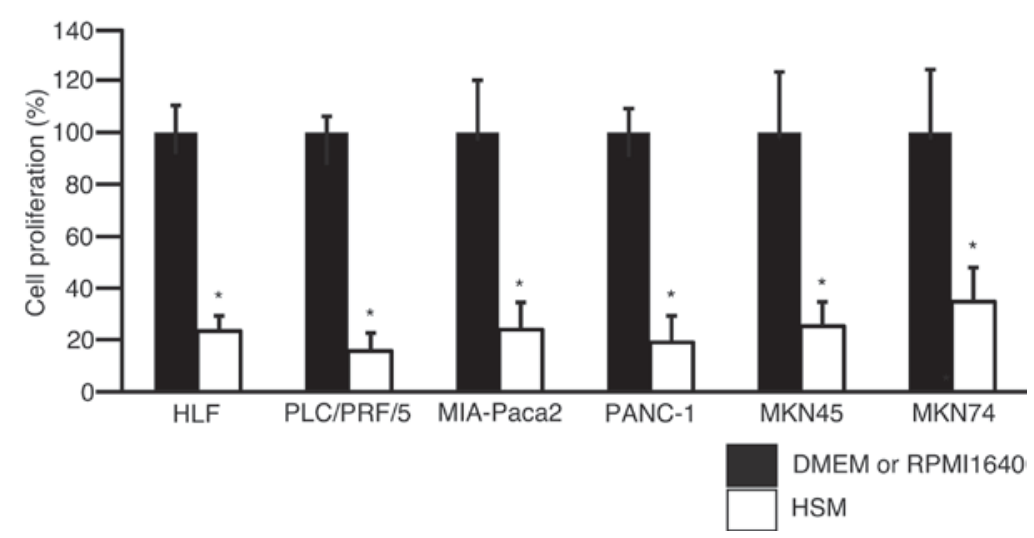

Figure 1. Cell proliferation suppressed by HSM. Each cell line was cultured in DMEM or RPMI-1640 supplemented with $10 \%$ fetal bovine serum or in HSM for three days. Data are presented as the mean \pm standard deviation. Error bar, standard deviation. "P $<0.05$, compared with culture in DMEM or RPMI-1640; n=3. DMEM, Dulbecco's modified Eagle's medium; HSM, hepatocyte selection medium.

was scratched and cultured in DMEM, RPMI-1640 or HSM for two days (Fig. 4A). The distances between the growing edge and the scratched edge were $5.4 \pm 1.0 \times 10^{2}$, $4.2 \pm 0.9 \times 10^{2}, 4.8 \pm 1.0 \times 10^{2}, 5.8 \pm 1.2 \times 10^{2}, 1.2 \pm 0.2 \times 10^{2}$ and $1.8 \pm 0.4 \times 10^{2} \mathrm{~mm}$, respectively, in HLF, $\mathrm{PLC} / \mathrm{PRF} / 5$, MIA-Paca2, PANC-1, MKN45 and MKN74 cells cultured in DMEM or RPMI-1640. The distances between the growing edge and the scratched edge were $4.3 \pm 0.5 \times 10^{2}, 7.7 \pm 1.3 \times 10$, $2.3 \pm 0.9 \times 10^{2}, 3.1 \pm 0.9 \times 10,3.0 \pm 0.8 \times 10$ and $1.5 \pm 0.3 \times 10^{2} \mathrm{~mm}$, respectively, in HLF, PLC/PRF/5, MIA-Paca2, PANC-1, MKN45 and MKN74 cells cultured in HSM. The distance between the growing edge and the scratched edge was observed as significantly decreased $(\mathrm{P}=0.03$ in HLF cells; $\mathrm{P}<0.01$ in $\mathrm{PLC} / \mathrm{PRF} / 5$, MIA-Paca2, PANC1, MKN45 and MKN74 cells) in HSM-cultured cells compared with cells cultured in DMEM or RPMI-1640 (Fig. 4B).

\section{Discussion}

In the present study, cell proliferation was significantly suppressed when cells were cultured in HSM, concordant with previous findings of reduced cell proliferation in a medium lacking glucose (23). The suppression of cell proliferation occurs in part due to cell cycle arrest (23).

Morphological analysis using hematoxylin and eosin staining suggested that the all the cell cultured in HSM had undergone apoptosis in the current study, which was further supported by the results of the TUNEL assay. Cancer cells primarily depend on glycolysis for energy (9); therefore, if glucose is lacking in the medium, cancer cells are unable to proliferate and undergo apoptosis (24). HSM does not contain glucose, and therefore, it is hypothesized that cancer cells grown in HSM undergo apoptosis due to glucose deprivation (25-27). In combination with previous findings, the results of the current study suggest that glucose metabolism may present a novel target for cancer treatment (28).

Arginine deprivation has also been demonstrated to trigger apoptosis in cancer cells, and is proposed as a novel approach for cancer treatment in melanoma and cervical cancer (29). One example is the use of arginase, which degrades arginine and induces apoptosis in cancer cells (30). A previous study indicated that cancer cells are resistant to arginine deprivation

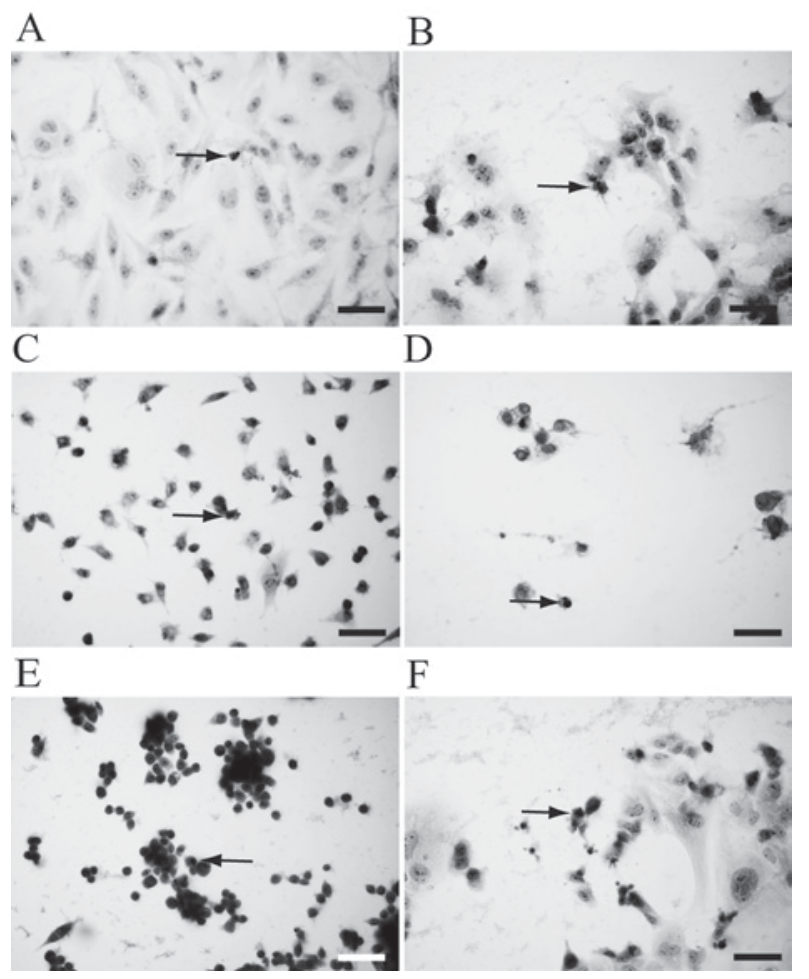

Figure 2. Apoptosis shown with hematoxylin and eosin staining in cells cultured HSM. Each cell line was cultured in HSM for two days and subjected to hematoxylin and eosin staining. Arrows indicate pyknotic nuclei. (A) HLF, (B) PLC/PRF/5, (C) MIA-Paca2, (D) PANC-1, (E) MKN45 and (F) MKN74 cells. Original magnification, $\mathrm{x} 400$; scale bar, $50 \mu \mathrm{m}$. HSM, hepatocyte selection medium.

in a three-dimensional environment (31). The current study used HSM, which does not contain glucose or arginine, and the results revealed that that deprivation of glucose and arginine may synergistically suppress cell proliferation and induce apoptosis. Therefore, targeting the metabolism of glucose and arginine appears to be a promising approach for cancer treatment.

In addition, the current study observed that the motility of cancer cells was suppressed in HSM. The effects of glucose or arginine deprivation on the motility of cancer cells remain to be elucidated; however, glucose deprivation is able to suppress 

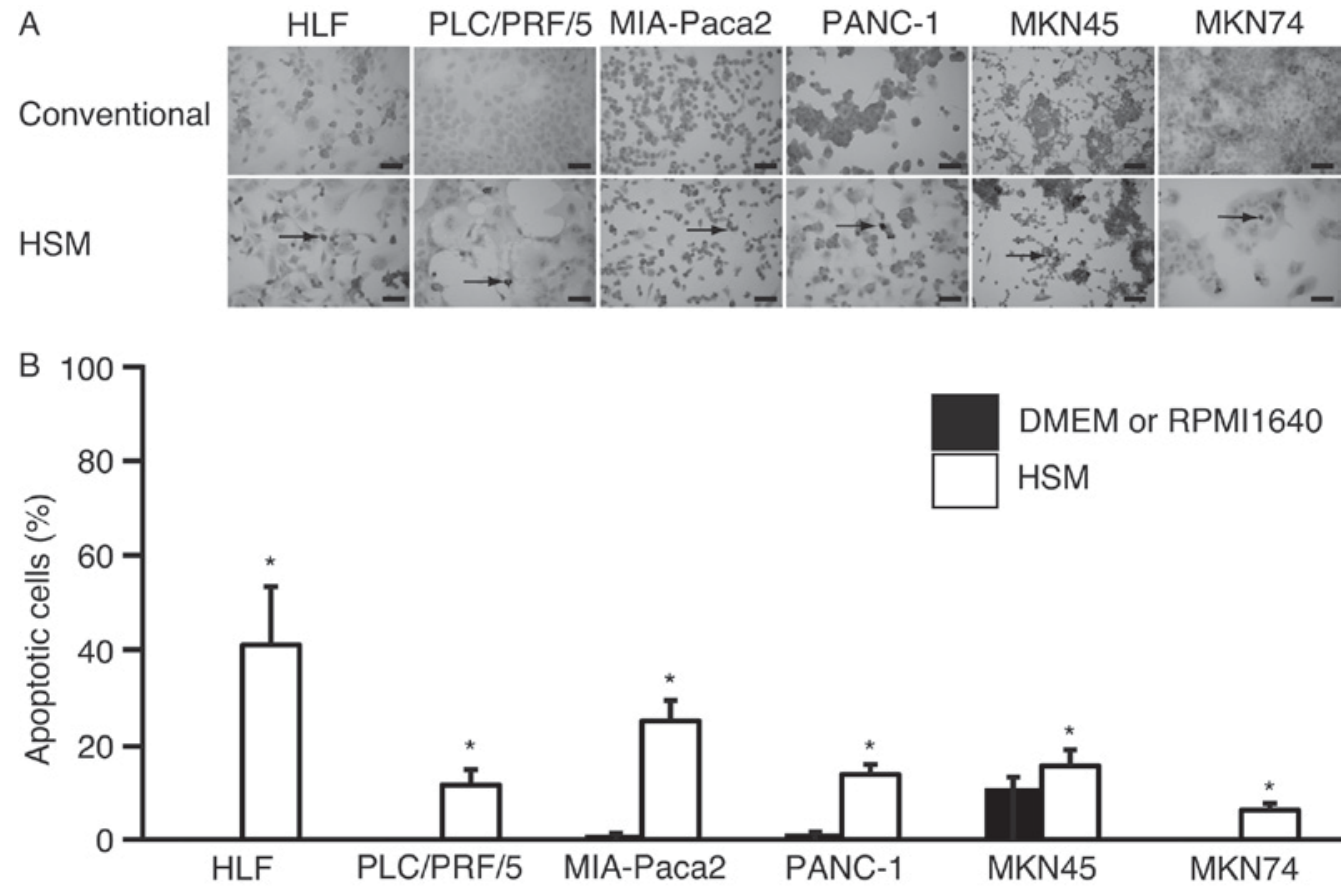

Figure 3. Ratios of apoptotic cells increased with hepatocyte selection medium. Results of the TUNEL assay. Each cell line was cultured in DMEM or RPMI-1640 supplemented with $10 \%$ fetal bovine serum, or in HSM for three days. (A) Images were captured (original magnification, x100; scale bar, $200 \mu \mathrm{m}$ ) for each cell line following the TUNEL assay. Arrows indicate TUNEL-positive cells. (B) Cells were counted and the proportion of apoptotic cells was calculated. Data are presented as the mean \pm standard deviation. Error bar, standard deviation. "P<0.05; $\mathrm{n}=3$. DMEM, Dulbecco's modified Eagle's medium; conventional, culture in DMEM or RPMI-1640 supplemented with 10\% fetal bovine serum; TUNEL, terminal deoxynucleotidyl transferase dUTP nick end labeling; HSM, hepatocyte selection medium.
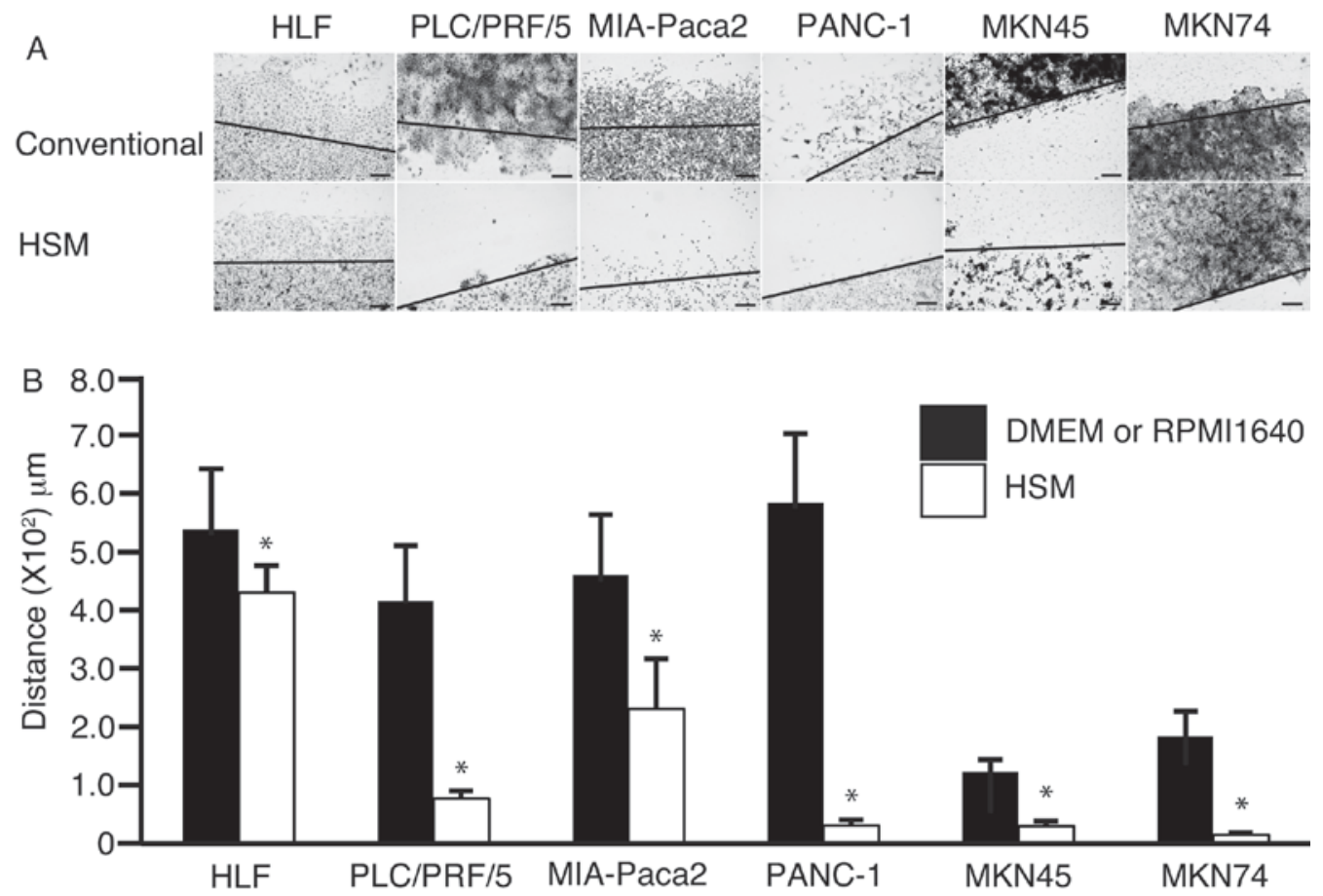

Figure 4. Cell motility suppressed in cells grown in HSM. (A) Each cell line was cultured in DMEM or RPMI-1640 supplemented with 10\% fetal bovine serum or hepatocyte selection medium following scratching of the cell monolayer. Original magnification, x100; scale bar, $200 \mu \mathrm{m}$. (B) The cells were subjected to hematoxylin and eosin staining after two days of culture, and cultured in DMEM or RPMI-1640 (closed bar), or hepatocyte selection medium (open bar) for two days. DMEM, Dulbecco's modified Eagle's medium; HSM, hepatocyte selection medium. $\mathrm{P}<0.05$ in cells cultured in HSM compared with those in DMEM or RPMI1640.

the motility of microglia in the mouse brain (32). Whether the results obtained for microglia have the same biological significance in cancer cells requires further study. However, the results of the current study suggested that the deprivation of glucose and arginine in combination inhibited the motility of cancer cells. 
An advantage of HSM culture is that it allows hepatocyte survival due to the presence of galactose and ornithine (21). Therefore, it was hypothesized in the present study that primary or metastatic liver cancer may be treated without associated hepatotoxicity by using HSM. Transcatheter arterial chemoembolization (TACE) is an established technique, whereas balloon occluding of the intrahepatic artery is a relatively novel technique that obstructs the artery with a micro-balloon and immerses the cancer cells in chemotherapeutic agents during TACE (33). Therefore, the current study hypothesizes that cancer cells may undergo apoptosis when cancerous tissues are immersed in HSM using balloon-occluded TACE.

In conclusion, the proliferation and motility of hepatocellular carcinoma cells, pancreatic cancer cells and gastric cancer cells were suppressed in a medium without glucose and arginine, but supplemented with galactose and ornithine (HSM). HSM may have potential as a new treatment of hepatocellular carcinoma.

\section{References}

1. Cong WM, Dong H, Tan L, Sun XX and Wu MC: Surgicopathological classification of hepatic space-occupying lesions: A single-center experience with literature review. World J Gastroenterol 17: 2372-2378, 2011.

2. Nakayama $\mathrm{H}$ and Takayama T: Role of surgical resection for hepatocellular carcinoma based on Japanese clinical guidelines for hepatocellular carcinoma. World J Hepatol 7: 261-269, 2015.

3. Petrelli F, Coinu A, Cabiddu M, Ghilardi M, Borgonovo K, Lonati $\mathrm{V}$ and Barni S: Hepatic resection for gastric cancer liver metastases: A systematic review and meta-analysis. J Surg Oncol 111: 1021-1027, 2015.

4. Lencioni R, Petruzzi P and Crocetti L: Chemoembolization of hepatocellular carcinoma. Semin Intervent Radiol 30: 3-11, 2013.

5. Kim HY and Park JW: Clinical trials of combined molecular targeted therapy and locoregional therapy in hepatocellular carcinoma: Past, present, and future. Liver Cancer 3: 9-17, 2014

6. Gnoni A, Santini D, Scartozzi M, Russo A, Licchetta A, Palmieri V, Lupo L, Faloppi L, Palasciano G, Memeo V, et al: Hepatocellular carcinoma treatment over sorafenib: Epigenetics, microRNAs and microenvironment. Is there a light at the end of the tunnel? Expert Opin Ther Targets 19: 1623-1635, 2015.

7. Raoul JL, Gilabert M and Piana G: How to define transarterial chemoembolization failure or refractoriness: A European perspective. Liver Cancer 3: 119-124, 2014.

8. Karczmarek-Borowska B and Sałek-Zan A: Hepatotoxicity of molecular targeted therapy. Contemp Oncol (Pozn) 19: 87-92, 2015.

9. Vaitheesvaran B, Xu J, Yee J, Q-Y L, Go VL, Xiao GG and Lee WN: The Warburg effect: A balance of flux analysis. Metabolomics 11: 787-796, 2015.

10. Gallamini A, Zwarthoed C and Borra A: Positron emission tomography (PET) in oncology. Cancers (Basel) 6: 1821-1889, 2014.

11. Gauthe M, Richard-Molard M, Cacheux W, Michel P, Jouve JL, Mitry E, Alberini JL and Lièvre A; Fédération Francophone de Cancérologie Digestive (FFCD): Role of fluorine 18 fluorodeoxyglucose positron emission tomography/computed tomography in gastrointestinal cancers. Dig Liver Dis 47: 443-454, 2015.

12. Leffert HL and Paul D: Studies on primary cultures of differentiated fetal liver cells. J Cell Biol 52: 559-568, 1972.

13. Matsumoto K, Yamada K, Kohmura E, Kinoshita A and Hayakawa T: Role of pyruvate in ischaemia-like conditions on cultured neurons. Neurol Res 16: 460-464, 1994.
14. Ohira RH, Dipple KM, Zhang YH and McCabe ER: Human and murine glycerol kinase: Influence of exon 18 alternative splicing on function. Biochem Biophys Res Commun 331: 239-246, 2005

15. Ai Y, Jenkins NA, Copeland NG, Gilbert DH, Bergsma DJ and Stambolian D: Mouse galactokinase: Isolation, characterization, and location on chromosome 11. Genome Res 5: 53-59, 1995.

16. Wheatley DN, Scott L, Lamb J and Smith S: Single amino acid (arginine) restriction: Growth and death of cultured HeLa and human diploid fibroblasts. Cell Physiol Biochem 10: 37-55, 2000.

17. Qiu F, Huang J and Sui M: Targeting arginine metabolism pathway to treat arginine-dependent cancers. Cancer Lett 364: $1-7,2015$

18. Phillips JW, Jones ME and Berry MN: Implications of the simultaneous occurrence of hepatic glycolysis from glucose and gluconeogenesis from glycerol. Eur J Biochem 269: 792-797, 2002.

19. Sumida KD, Crandall SC, Chadha PL and Qureshi T: Hepatic gluconeogenic capacity from various precursors in young versus old rats. Metabolism 51: 876-880, 2002.

20. Tomizawa M, Toyama Y, Ito C, Toshimori K, Iwase K, Takiguchi M, Saisho $\mathrm{H}$ and Yokosuka O: Hepatoblast-like cells enriched from mouse embryonic stem cells in medium without glucose, pyruvate, arginine, and tyrosine. Cell Tissue Res 333: 17-27, 2008.

21. Tomizawa M, Shinozaki F, Sugiyama T, Yamamoto S, Sueishi M and Yoshida T: Survival of primary human hepatocytes and death of induced pluripotent stem cells in media lacking glucose and arginine. PLoS One 8: e71897, 2013.

22. Nakamura T, Teramoto H, Tomita Y and Ichihara A: L-proline is an essential amino acid for hepatocyte growth in culture. Biochem Biophys Res Commun 122: 884-891, 1984.

23. Fu P, Tang R, Yu Z, Huang S, Xie M, Luo X and Wang W: Bumetanide-induced NKCC1 inhibition attenuates oxygen-glucose deprivation-induced decrease in proliferative activity and cell cycle progression arrest in cultured OPCs via P-38 MAPKs. Brain Res 1613: 110-119, 2015.

24. Gonzalez CD, Alvarez S, Ropolo A, Rosenzvit C, Bagnes MF and Vaccaro MI: Autophagy, Warburg, and Warburg reverse effects in human cancer. Biomed Res Int 2014: 926729, 2014.

25. Qiu J, Zhou XY, Zhou XG, Li Y, Cheng R and Liu HY: MicroRNA210 knockdown contributes to apoptosis caused by oxygen glucose deprivation in PC12 cells. Mol Med Rep 11: 719-723, 2015.

26. Wardi L, Alaaeddine N, Raad I, Sarkis R, Serhal R, Khalil C and Hilal G: Glucose restriction decreases telomerase activity and enhances its inhibitor response on breast cancer cells: Possible extra-telomerase role of BIBR 1532. Cancer Cell Int 14: 60, 2014.

27. Kim HS, Kim MJ, Lim J, Yang Y, Lee MS and Lim JS: NDRG2 overexpression enhances glucose deprivation-mediated apoptosis in breast cancer cells via inhibition of the LKB1-AMPK pathway. Genes Cancer 5: 175-185, 2014.

28. Elf SE and Chen J: Targeting glucose metabolism in patients with cancer. Cancer 120: 774-780, 2014.

29. Feun LG, Kuo MT and Savaraj N: Arginine deprivation in cancer therapy. Curr Opin Clin Nutr Metab Care 18: 78-82, 2015.

30. Zeng X, Li Y, Fan J, Zhao H, Xian Z, Sun Y, Wang Z, Wang S, Zhang $G$ and Ju D: Recombinant human arginase induced caspase-dependent apoptosis and autophagy in non-Hodgkin's lymphoma cells. Cell Death Dis 4: e840, 2013.

31. Vynnytska-Myronovska B, Kurlishchuk Y, Bobak Y, Dittfeld C, Kunz-Schughart LA and Stasyk O: Three-dimensional environment renders cancer cells profoundly less susceptible to a single amino acid starvation. Amino Acids 45: 1221-1230, 2013.

32. Eyo U and Dailey ME: Effects of oxygen-glucose deprivation on microglial mobility and viability in developing mouse hippocampal tissues. Glia 60: 1747-1760, 2012.

33. Imai N, Ishigami M, Ishizu Y, Kuzuya T, Honda T, Hayashi K, Hirooka Y and Goto H: Transarterial chemoembolization for hepatocellular carcinoma: A review of techniques. World J Hepatol 6: 844-850, 2014. 